\title{
Computer-aided design of horizontal axis turbine blades
}

\author{
F. Pérez-Arribas, I. Trejo-Vargas \\ Universidad Politécnica de Madrid. (UPM), Avenida Arco de la Victoria SN., 28040 Madrid, \\ Spain
}

A B S T R A C T

This paper presents a computer-based method for modelling the blades of horizontal axis turbines using B-spline surfaces. The method uses common design parameters for the geometry of this type of turbine and produces a final set of B-spline surfaces for the geometry of the blades that can be used for the visualisation, calculations and construction of the rotor surface. The method begins with the creation of a 3D set of offsets that constitute the rotor blades based on a $2 \mathrm{D}$ definition of the airfoils, which is normally used in the design of different stations along the rotor blade. It also uses geometrical param-eters such as the skew and rake or coning distribution. The method stresses the fitting of the blade's leading edge, which has a significant impact on the properties of the rotor and separately models the trailing edge of the blades. B-spline curves and surfaces are used in this method because they are widely used in CAD-CAM software products and can be easily exported to other programs.

\section{Introduction}

A horizontal axis rotor consists of a number of identical twisted blades spaced equally around a hub or boss. The blade shape is defined by a series of airfoil sections at specified radius ratios of the rotor, and these sections can be circular when they are close to the hub so that the blade can be pitched around. The inclination of the blades from the rotor plane is given by the rake or coning of the sections.

A typical left-hand rotor is presented in Fig. 1, in which the rotor plane is the $X Z$ plane and the flow direction is along the $Y$ axis, which is also the rotation axis. In this figure, the rotor hub is omitted.

The blade design and its geometrical definition are intrinsically related to the selection of a $2 \mathrm{D}$ airfoil and its geometrical characteristics considering both their aerodynamic or hydrodynamic performance and their stiffness for large rotors. Therefore, the geometrical characteristics of the airfoils must be accurately modelled in a $3 \mathrm{D}$ definition of the rotor blades.

The approach presented for defining the geometry of the rotor blades is based on a discrete approximation and interpolation of a collection of space curves, which are also called cross sections, containing a 3D representation of the 2D airfoil points. The manner in which these points are obtained and the tolerances associated

\footnotetext{
* Corresponding author.

E-mail address: francisco.perez.arribas@upm.es (F. Pérez-Arribas).
}

with the data, influence the presented method for the representation of the blades.

Among various mathematical surface representations, B-splines and NURBS (non-uniform rational B-splines) have become industry standards, and many engineering products such as FEA (Finite Element Analysis), CFD (Computational Fluid Dynamics) and manufacturing softwares use these types of surfaces to conduct their calculations.

However, modelling the geometry of rotor blades is different from modelling other industrial objects; for example, an accurate representation of the shape of the airfoils is required, and this requires working with a large amount of information because the representation of these curves is based on a discrete set of data points, usually more than 100 points per airfoil. If standard techniques are used, very complex surfaces with a large number of control points are obtained and will likely present poor smoothness in the resulting surface obtained from an approximation of the interpolation of the data sets.

These problems are difficult to solve by direct manipulation of the control points in the surface or surfaces, although different techniques can be used $([1,2])$ for convenient shape modifications of the surfaces.

A constraint of the present problem is that the leading edge of the blades must be accurately reproduced by the surface because most of the aerodynamic characteristics are induced by this region. This part of the surface presents high curvature values when compared with the rest of the shape of the blades (see Fig. 6 in Section 4). A solution for this area is included in the method and is presented in detail in Section 3. 


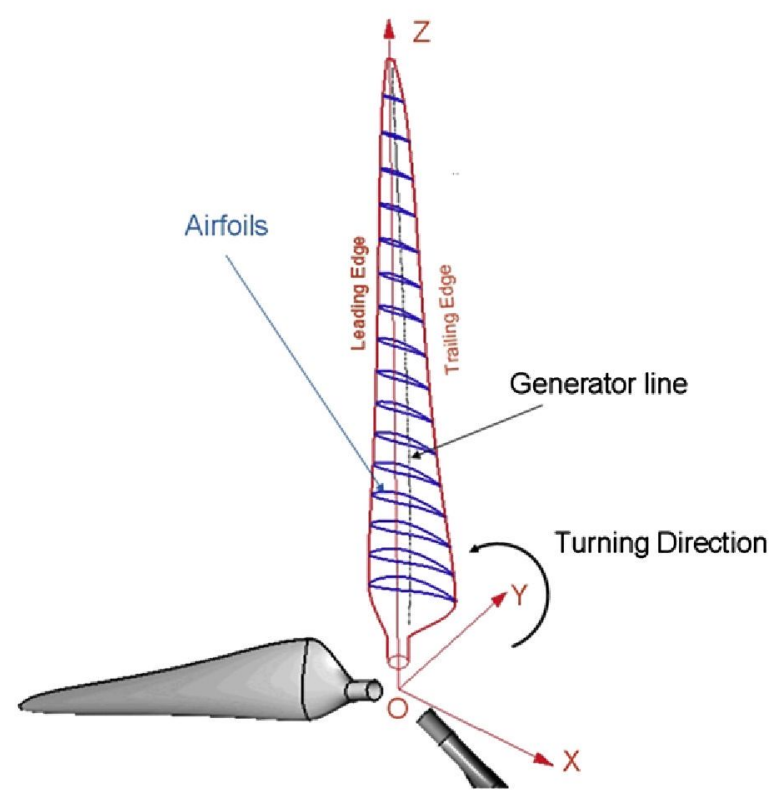

Fig. 1. Rotor geometry.

The technique of lofting or skinning over B-spline curves was selected from the various numerical methods used for industrial surface definitions. This consists of fitting a surface through an ordered set of cross-sectional curves $([3,4])$, which in this case are airfoil curves. Accurate modelling of the 3D points corresponding to the airfoils is important to the success of the method.

A least squares (LS) fitting process ([5]) with an original selection of the parameterisation based on the Haussdorf metric (the minimum of the maximum Euclidean distances) is described in Section 3 and also considers accurate modelling of the leading edge. The method also defines a rounded trailing edge of the sections, which is normally modelled as a single point, considering its constructability.

The proposed method allows for a reduction in the number of control points of the surface without reducing accuracy. Data reduction in the surface representation speeds up most of the downstream processes, improves the fairing of the resulting surface and decreases storage requirements in following design stages.

There are a few references that relate directly to model rotor blades. Ref. [6] also used the lofting of airfoils to model rotor blades but did not explain how to deal with the airfoil data points. Rather, it focused on the lofting procedure over profile curves on concentric cylinders. The authors did not use tolerances in their work or consider the trailing edge.

Ref. [7] proposed a method that included lofting but interpolated the data points instead of approximating them. They modified the data points to produce more points in the leading edge, whereas the presented method does not alter the data points. Their method was applied for air wings that did not present any twist.

An interesting reference is [8], which focused on aerodynamic airfoil optimisation and used B-spline curves for its definition. The examples in that work are compared with real airfoil shapes in
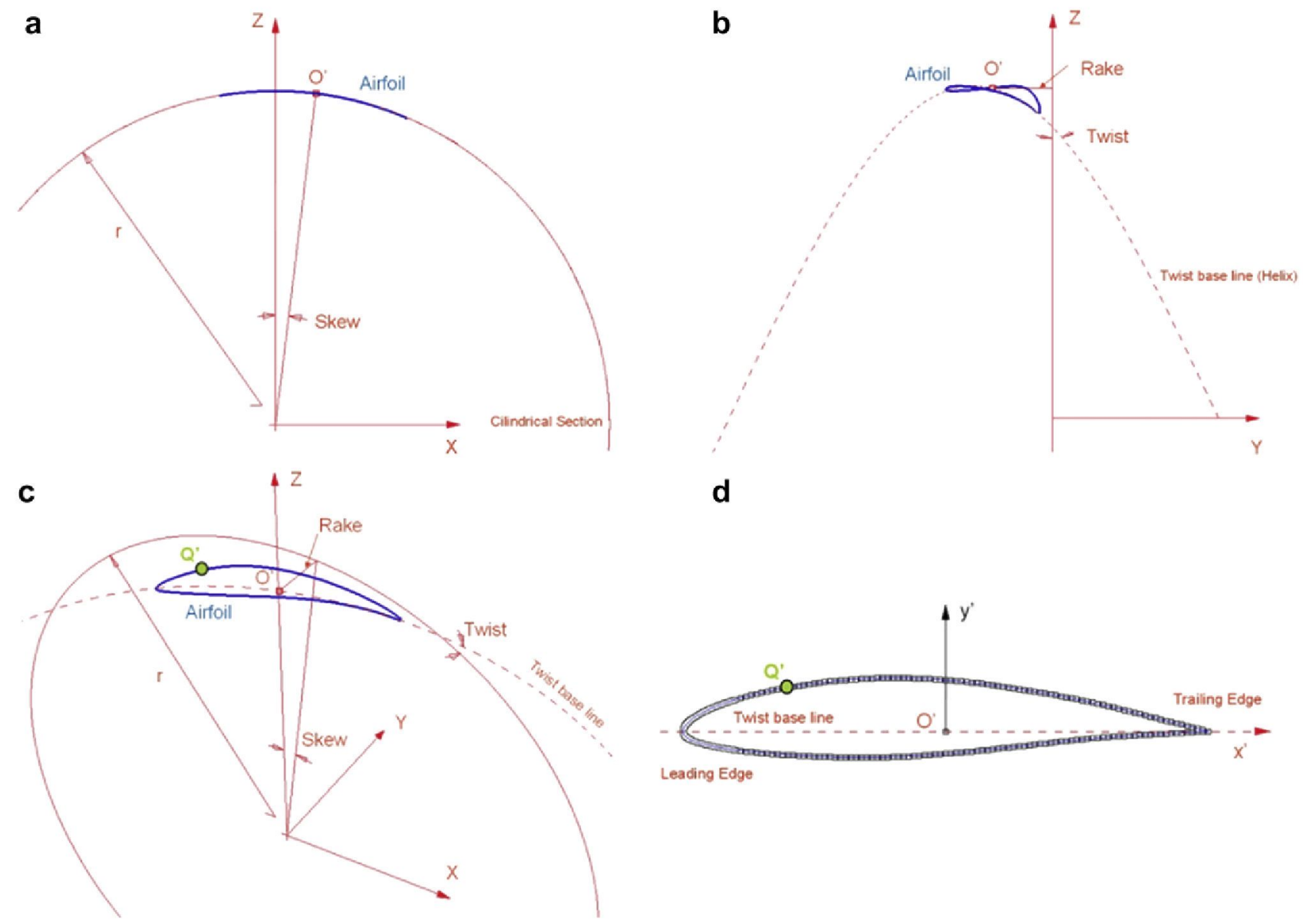

d

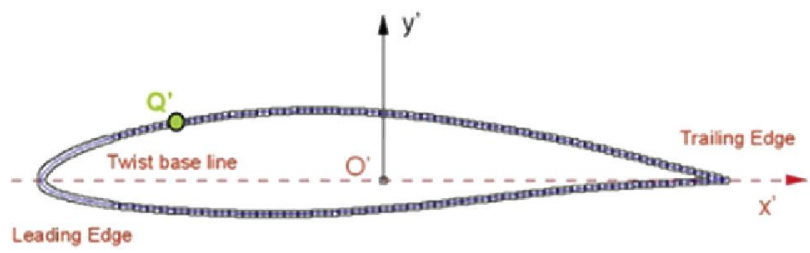

Fig. 2. Geometry of the airfoil sections. 


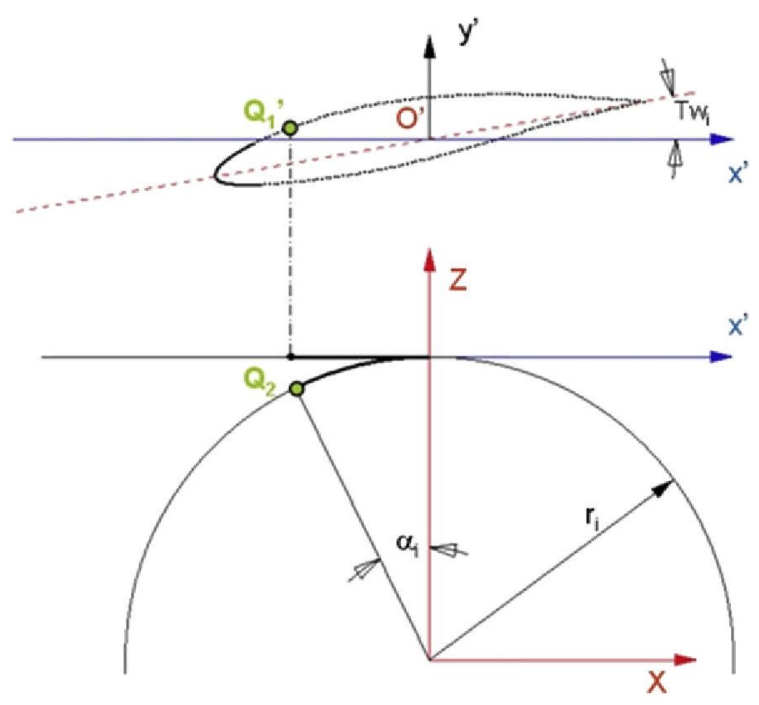

Fig. 3. Twisting and bending.

Section 4. That work began with a given definition of the control points of a B-spline that models the $2 \mathrm{D}$ airfoil data points and optimises the position of the control points until a given tolerance is reached. The presented method in this paper does not require an initial B-spline for modelling the airfoil points.

The method outline follows; first, the presented method reads the geometric information of the rotor blades, including the position, skew, rake and twist distribution and the discrete data definition of the sections, which the designer has determined after the initial design stage. Then, a discrete 3D definition of the airfoils is defined, and a curve fitting scheme is applied to the discrete data to define the $3 \mathrm{D}$ airfoils, resulting in B-spline curves with their respective control points. Finally, a lofting surface of the previous curves is applied, following the span-wise direction along which the airfoil sections are positioned. Two application examples of rotors are used to evaluate the practical usage of the presented method. An annex with the notation for this paper and a brief discussion of B-spline curves is at the end of this work.

The objective with this paper is to create a practical method to define the surface of turbine blades, according to parameters that are related with its performance. This parameters can be selected and optimized with the used of a BEM (Blade Element Method) theory, and once that a set of surfaces for the blades are defined, optimization algorithms can be used for optimization together with CFD (Computational Fluid Dynamic) calculations.

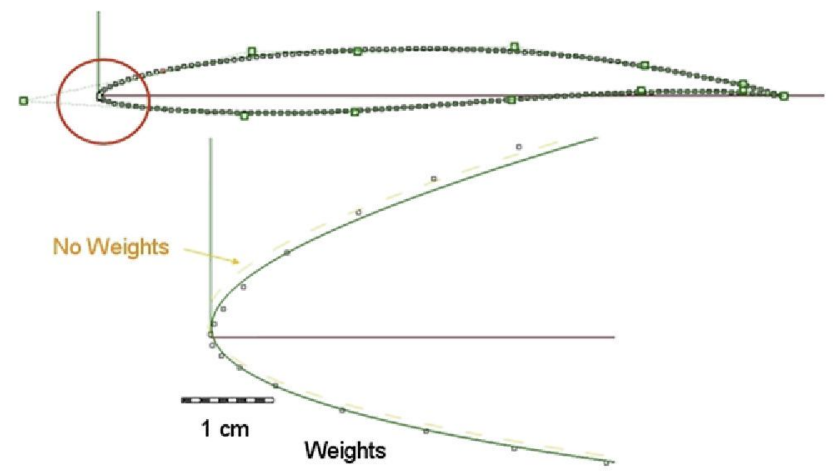

Fig. 4. Effect of weights on the leading edge of MH121 with 13 control points.

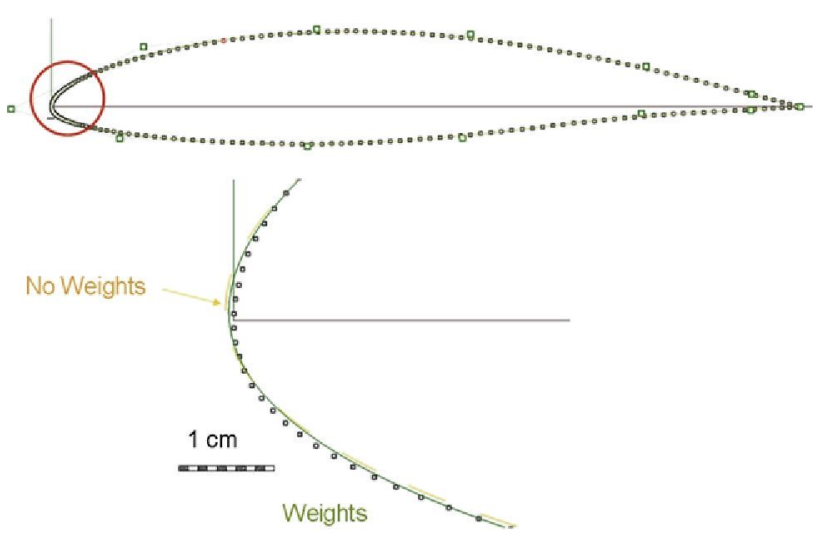

Fig. 5. Effect of weights on the leading edge of NACA 642415 for 13 control points.

There are several good textbooks and references, see e.g. [9-11] that show the effect of most of the parameters that the presented method uses (that are of common use in blade turbine design), into the performance of a turbine. It is not the goal of the paper to create an optimised design from performance point of view, but to enable a parametric definition and a complete generation of a turbine rotor, which allows the designer the selection of the parameters' values under performance considerations.

\section{Discrete surface definition}

The first step is to create a 3D definition of the airfoils so they can be fitted with B-spline curves. Considering the same references as in Fig. 1, the base line of each airfoil section is part of a helix line,as depicted in Fig. 2b) and c) and Fig. 3 which winds on the surface of a cylinder of radius $r$. The twist angle varies along the span of the blade, and the twist is defined as the angle between the airfoil base line and the circle of radius $r$ inside the rotor plane that corresponds to the section, as shown in Fig. 2c). This circle is also the intersection of a cylinder of radius $r$ that contains the airfoil with the $X Z$ rotor plane.

The rake is defined for each section as the distance from the airfoil origin $O^{\prime}$ to the rotor plane, as depicted in Fig. 2b) and c). The rake of each section can be defined as a function of a constant angle for the whole blade (rake or coning angle), so the rake of each section is a linear function of this angle.

The skew angle is the angle between $O^{\prime}$ and the $Z$ axis, and it controls the shape of the blade, as shown in Fig. 2a) and c). This angle is obtained based on the generator line that crosses through the reference points $O^{\prime}$ of the sections, as shown in Fig. 1. It can be a straight line or a soft curve according the design characteristics.

The origin $O^{\prime}$ of the airfoils can be placed well within the chord midpoint (Fig. 2d), at the point of maximum thickness or even at the leading edge. Therefore, its position affects the value of the skew distribution.

All of the above-mentioned geometric parameters, together with the number of blades, diameter and offset tables to define the airfoil sections at specified radii, are required to define the geometry of the rotor. These data are used to generate the $3 \mathrm{D}$ coordinates of points contained on the surfaces of the blades placed at different radius ratios (stations). In this way, the coordinate points of the surface of a single blade are obtained. The rest of the blades are formed by a homogeneous coordinate transformation of a single blade about the axis of rotation. The hub is modelled in its simplest form as the frustum of a right circular cone. 

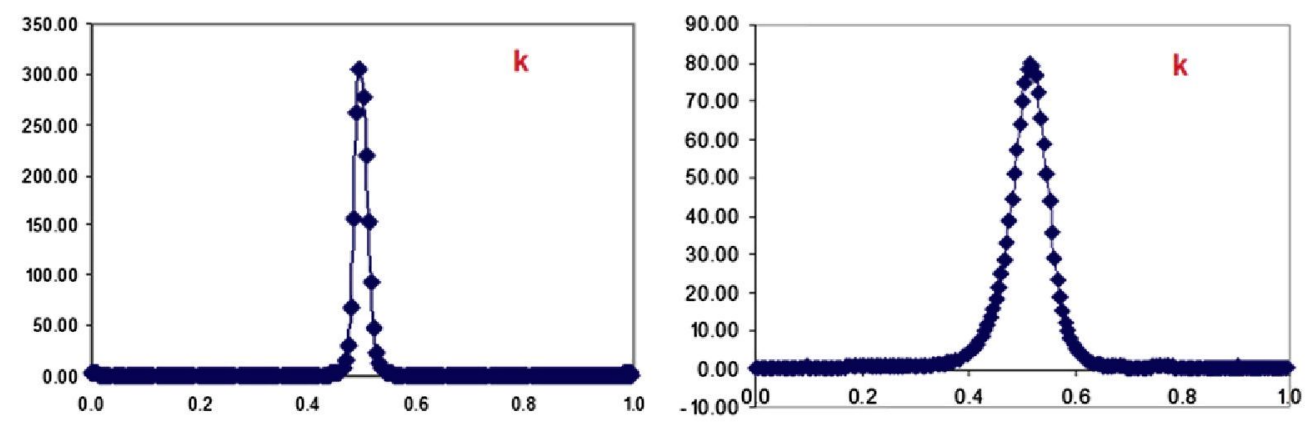

Fig. 6. Curvatures for MH121 and NACA 642415.

The 3D coordinates of any point $\boldsymbol{Q}_{\boldsymbol{j}}(x, y, z)$, as shown in Fig. 2c), can be obtained based on their associated 2D coordinates $\boldsymbol{Q}_{j}^{\prime}\left(x^{\prime}, y^{\prime}\right)$ following these steps:

1) The twist angle of each section $\mathrm{Tw}_{i}$ is applied, and $\boldsymbol{Q}_{1}^{\prime}$ is obtained:

$R=\sqrt{x^{2}+y^{\prime 2}}$

$\theta=$ at $\operatorname{an}\left(\mathrm{y}^{\prime} / \mathrm{x}^{\prime}\right)$

$\mathbf{Q}_{1}^{\prime}=\left(R \cdot \operatorname{Cos}\left(\theta+\mathrm{Tw}_{i}\right), R \cdot \operatorname{Sin}\left(\theta+\mathrm{Tw}_{i}\right)\right)$

2) The point $\mathbf{Q}_{1}^{\prime}$ is rolled over the surface of the cylinder or radius $r_{i}$ of the $i$ th section to obtain $\mathbf{Q}_{2}$. This bending sets the arc length over the cylinder to be equal to the abscissa of $\boldsymbol{Q}_{1}^{\prime}$ :

$\alpha_{i}=\frac{x^{\prime}\left(\boldsymbol{Q}_{1}^{\prime}\right)}{r_{i}}=\frac{R \cdot \operatorname{Cos}\left(\theta+\mathrm{Tw}_{\boldsymbol{i}}\right)}{r_{i}}$

$\mathbf{Q}_{2}=\left(r_{i} \cdot \operatorname{Sin}\left(\alpha_{i}\right), \quad R \cdot \operatorname{Sin}\left(\theta+\mathrm{Tw}_{i}\right), r_{i} \cdot \operatorname{Cos}\left(\alpha_{i}\right)\right)$

3) The final step applies the skew $\left(\mathrm{Sk}_{i}\right)$ and rake $\left(\mathrm{Rk}_{i}\right)$ to the bent points by adding these magnitudes to the angle $\alpha_{i}$ and to the Yth coordinate, respectively. These additions maintain the points over the surface of the cylinder of radius $r_{i}$ :

$\mathbf{Q}_{\boldsymbol{j}}=\left(r_{i} \cdot \operatorname{Sin}\left(\alpha_{i}+\mathrm{Sk}_{i}\right), \quad R \cdot \operatorname{Sin}\left(\theta+\mathrm{Tw}_{i}\right)+\mathrm{Rk}_{\boldsymbol{i}}, \quad r_{i} \cdot \operatorname{Cos}\left(\alpha_{i}+\mathrm{Sk}_{i}\right)\right)$

By repeating this procedure for all points $(j=0, n p(i))$ of each airfoil $(i=0, M)$, a discrete approximation of the blades by a set of data points is obtained. The next step is the modelling of these discrete curves as B-splines so the final lofting can be obtained.

\section{Definition of the airfoil B-splines}

Once the np $(i)+1$ discrete data points $\boldsymbol{Q}_{\boldsymbol{j}}$ have been obtained, the B-spline fitting can be conducted. Although the number of points $n p(i)$ can vary for each airfoil, in this section it is cited as np for the sake of clarity. This set of np points is based on $2 \mathrm{D}$ points as detailed in the previous section. The $2 \mathrm{D}$ data can be generated by the computer program used for designing the rotor, they can be calculated analytically in the case of NACA airfoils, or they can be read from a database library; therefore, these data are subject to measurement errors and noise. These points can be uniformly distributed or present a higher density in the leading edge area depending on the source of the data.

A large number of data points do not suggest the use of an interpolating B-spline. Instead, an approximating curve is required. For each airfoil curve of index " $d$ ", the B-spline $\boldsymbol{c}_{\boldsymbol{d}}(\boldsymbol{u})$ does not pass through the data points exactly but passes close enough to the points to capture their inherent shape. This is the well-known least squares (LS) approximation, which is discussed in [5].

For this problem, $n p+1$ data points $\mathbf{Q}_{0}, \ldots \mathbf{Q}_{\mathbf{n p}}$ are approximated by a B-spline of the $p$ th degree with $N+1$ control points $\boldsymbol{P}_{\mathbf{0}}, \ldots \boldsymbol{P}_{N}$, $N<$ np that are unknown and are obtained as the final results of the calculations.

The general LS problem is described by an over-determined set of np +1 equations with $N+1$ unknown variables:

$\mathrm{B}_{0}^{p}\left(t_{0}\right) \cdot P_{0}+\mathrm{B}_{1}^{p}\left(t_{0}\right) \cdot P_{1}+\ldots+\mathrm{B}_{N}^{p}\left(t_{0}\right) \cdot P_{N}=Q_{0}$
$\mathrm{~B}_{0}^{p}\left(t_{1}\right) \cdot P_{0}+\mathrm{B}_{1}^{p}\left(t_{1}\right) \cdot P_{1}+\ldots+\mathrm{B}_{N}^{p}\left(t_{1}\right) \cdot P_{N}=Q_{1}$
$M \quad M \quad M \quad M$
$\mathrm{~B}_{0}^{p}\left(t_{\mathrm{np}}\right) \cdot P_{0}+\mathrm{B}_{1}^{p}\left(t_{\mathrm{np}}\right) \cdot P_{1}+\ldots+\mathrm{B}_{N}^{p}\left(t_{\mathrm{np}}\right) \cdot P_{N}=Q_{\mathrm{np}}$

where $\mathrm{B}_{i}^{p}$ corresponds to the $i$ th basis function of a $p$ th degree $\mathrm{B}-$ spline that is calculated using de Boor's algorithm in Eq. (11) considering a uniform knot vector, and $t_{j}(j=0, n)$ represents the parameters associated with the data points. Matrix expressions are convenient to solve the problem:

$$
\begin{aligned}
& {\left[\begin{array}{cccc}
\mathrm{B}_{0}^{p}\left(t_{0}\right) & \mathrm{B}_{1}^{p}\left(t_{0}\right) & \ldots & \mathrm{B}_{N}^{p}\left(t_{0}\right) \\
\mathrm{B}_{0}^{p}\left(t_{1}\right) & \mathrm{B}_{1}^{p}\left(t_{1}\right) & \ldots & \mathrm{B}_{N}^{p}\left(t_{1}\right) \\
M & M & M & M \\
\mathrm{~B}_{0}^{p}\left(t_{\mathrm{np}}\right) & \mathrm{B}_{1}^{p}\left(t_{\mathrm{np}}\right) & \ldots & \mathrm{B}_{N}^{p}\left(t_{\mathrm{np}}\right)
\end{array}\right] \cdot\left|\begin{array}{c}
P_{0} \\
P_{1} \\
M \\
P_{N}
\end{array}\right|=\left[\begin{array}{c}
Q_{0} \\
Q_{1} \\
M \\
Q_{n p}
\end{array}\right] ;[M] \cdot[P]} \\
& =[Q] \Rightarrow[M]^{T} \cdot[M] \cdot[P]=[M]^{T} \cdot[Q]
\end{aligned}
$$

Weighted fitting : $[M]^{T} \cdot[M] \cdot[w] \cdot[P]=[M]^{T} \cdot[w] \cdot[Q]$;

This system of equations is solved by multiplying both sides of Eq. (5) by $[M]^{T}$, which creates a determined $(N+1)$ by $(N+1)$ linear system. The standard LS is modified using weights because of the aspect of the airfoil data points; the leading edge produces a high curvature zone (Fig. 6) and a standard LS does not fit this part of the curve with enough accuracy unless a large number of control points are used. These weights affect the points of the leading edge, increasing the fitting accuracy in this area, which has a major effect on the performance of the blade.

This type of system can be poorly conditioned, especially if a large number of control points are used. A conventional technique should not be used to solve this ill-conditioned system. Instead, a single-value decomposition of $[M]^{T}[M]$ and a later backsubstitution process are performed. The solutions of this system are the control points of the best B-spline fitting. 
Approaching this problem with a standard parameterisation such as centripetal or chord-length parameterisation is correct but does not consider the effect of the distance of the data points to the $\mathrm{B}$-spline. In this method, parameterisation based on a minimum distance metric is adopted. The process is iterative and is described by the following three steps:

1. The method begins with a centripetal parameterisation of the $\mathbf{Q}_{i}$ points, and system (5) is solved. This produces a starting curve of the iterative process only for the first loop.

2. For each $\boldsymbol{Q}_{i}$, the minimum distance to the B-spline is calculated. This is accomplished by dividing the B-spline $\boldsymbol{c}_{\boldsymbol{d}}(\boldsymbol{u})$ into Bézier curves $\boldsymbol{b}_{j}\left(\boldsymbol{u}_{L}\right)(j=1, N-p)$ of the $p$ th degree and computing the minimum distance to the corresponding Bézier piece, which leads to a solution to Eq. (6).

$$
\left(\mathbf{Q}_{\boldsymbol{i}}-\boldsymbol{b}_{\boldsymbol{j}}\left(u_{L}\right)\right) \cdot\left(\boldsymbol{b}_{j}^{\prime}\left(u_{L}\right)\right)=0
$$

This equation is solved in the local domain of the Bézier curves. Because the equation is a polynomial equation, $u_{L} \in[0,1]$, specific algorithms for this type of equation can be used. These algorithms do not require an initial guess, which would be required if a Newtonian method were used in the B-spline domain. The current method uses a Jenkins-Traub 3-stage algorithm [12]. The valid solution is a non-complex solution of $u_{L} \in[0,1]$.

Once the solution has been found, the local $u_{L}$ for the Bézier domain is easily converted into its global value $t_{i}$ in the B-spline domain. This $t_{i}$ value is the parameter associated with the point $\mathbf{Q}_{i}$ when solving system (5).
3. After obtaining the $t_{i}(i=1, \mathrm{np})$ values, the distance $d_{i}=\left(\boldsymbol{Q}_{\boldsymbol{i}}-\boldsymbol{c}_{\boldsymbol{d}}\left(t_{i}\right)\right)$ is computed, which is the Euclidean distance between $Q_{i}$ and the B-spline. This distance is used to verify the shape requirements. If the maximum distance $d_{i}(i=1, n)$ is above a given tolerance, steps 2 and 3 are repeated until an acceptable maximum distance is obtained. The quality of the obtained curve is measured using the tolerance constraint, and the shape of the B-spline is amended using parameterisation (6).

If the tolerance is not obtained in fewer than 50 iterations, then the number of control points $N+1$ has to be increased. An increment in the degree $p$ in this procedure can also reduce the maximum distance. However, increasing the number of control points has a more substantial effect, and a higher degree increases the complexity and the computation time.

\section{Evaluation of the fitting}

This section presents some cases of the previously described fitting by comparing the present method with the examples shown in $[8,13]$, which correspond to real airfoil sections. The examples in Table 1 present different shapes to give relevant results for real airfoils assuming a common chord of $1 \mathrm{~m}$.

The above-mentioned references used a B-spline technique for optimisation purposes and controlled the shape of the airfoil by moving its control points and optimising aerodynamic properties under certain constraints. These authors used the airfoils of

Table 1

Airfoils tested.

MH126 (Thick) $t / c=25.1 \%$

MH121 (Thin \& Cambered) $t / c=8.8 \%$

MH43 (Thin) $t / c=8.5 \%$
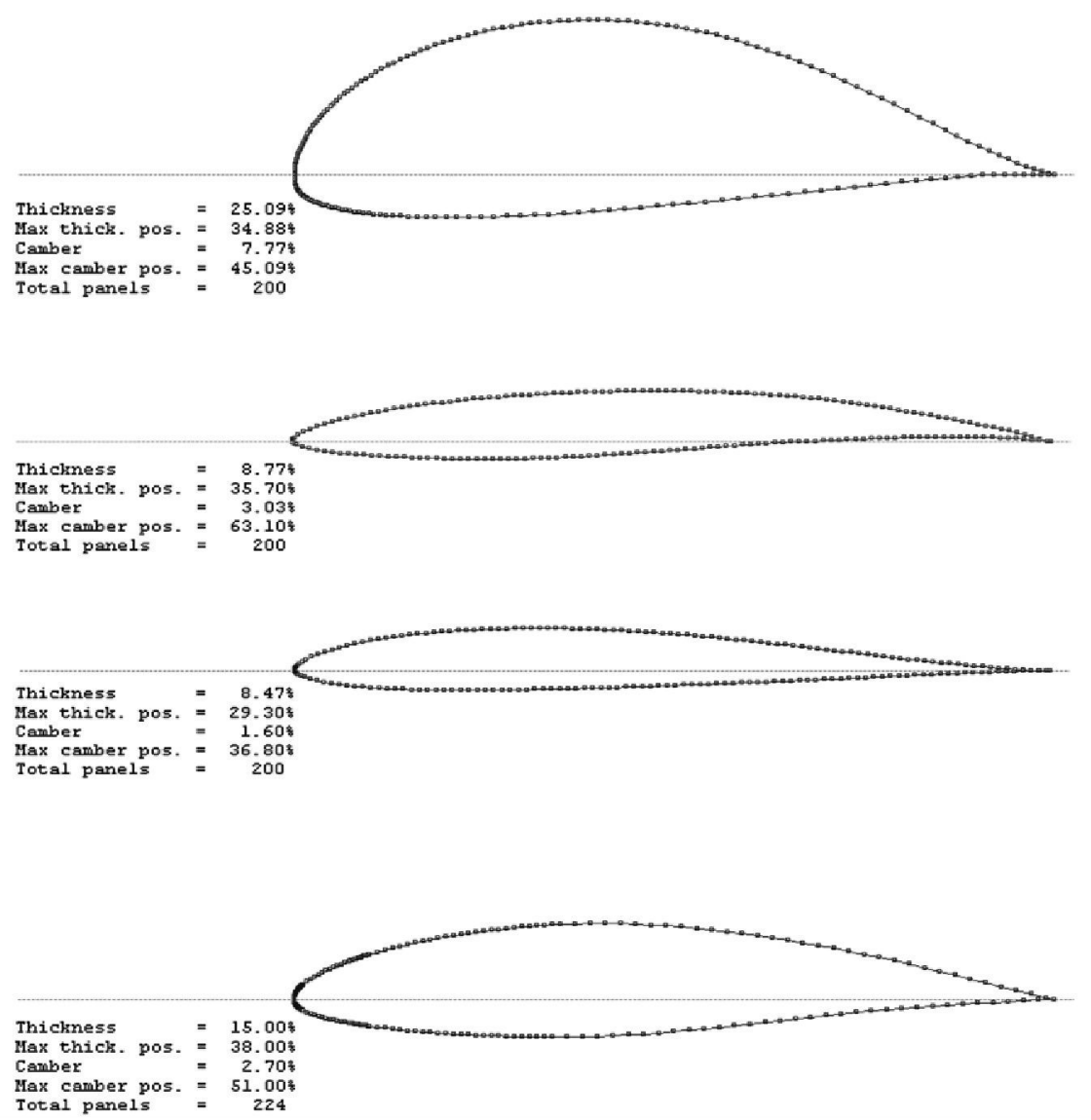
Table 1 as reference examples, but the manner in which they fit the data points was different from that presented in this paper; an initial B-spline was required as an initial guess, and the positions of its control points (only their ordinates according the references) were numerically modified until a given tolerance was reached.

The effects of the weights are shown in Figs. 4 and 5, in which 200 points of the MH121 airfoil were fitted to a cubic B-spline with just 13 control points. The positions of the control points are plotted in the upper part of the figure, and the details of the leading edge are also shown.

A similar figure shows the fitting for the Naca profile, which also considered 200 points and 13 control points. In this case, the point density in the leading edge was higher than in the previous profile, which is positive for the fitting that did not consider weights.

The weights of Eq. (5) were selected as 3 for the points that present a curvature higher than $10 \%$ of the highest curvature. Curvature plots for the examples of the previous figures are displayed in Fig. 6. The aspect of the curvature $k$ distribution is common for any airfoil; a peak value in the leading edge occurs at approximately $u=0.5$ when the points are fitted with a B-spline.

If the high curvature of the leading edge is not considered, then the fitting in this area is not as good as in the rest of the curve, especially when a low number of control points are used and the point density in this area is small. Although the fitting of the leading edge is always better when using the proposed weight distribution, the fitting in the rest of the curve may be slightly worse, as shown in Table 2, when comparing the maximum deviation. This is also a function of the distribution of the data points.

When using the method presented for fitting, the maximum and average distances between the B-spline and the data points can be computed. For the tested cases shown in Table 1 , the maximum deviation between the data points and the B-spline is presented in metres in columns named "Weights" and "No Weights", and compared with the values presented in $[8,13]$ that appear in columns 11 CPs $\sim 14$ CPs.

The calculations for 11,12 and 13 control points correspond to [13], and the calculations for 14 control points correspond to [8]. The data point distribution is not the same as the one used in the Refs. $[8,13]$ because the original data points were not presented in the references, but the number of data points was 200 in all of the cases. Note that the deviations are of the same order of magnitude in all of the cases and that the presented method improves the results for thicker profiles (MH126 and Naca). The maximum deviation can be reduced by increasing the number of control points. For the examples in Table 1 , a tolerance below $0.001 \mathrm{~m}$ was reached with 21 control points.

Most wind turbines possess circular sections on their blade root near the hub to change the blade pitch. These circular sections are not a problem for the presented fitting approach because they can be treated as airfoils; the circle is divided into a set of points, their

Table 2

Maximum deviations of the examples (metres) for different number of control points (CPs).

\begin{tabular}{lllllll}
\hline & 11 CPs & Weights & No. weights & 12 CPs & Weights & No. weights \\
\hline MH126 & 0.0052 & 0.0023 & 0.0020 & 0.0054 & 0.0013 & 0.0012 \\
MH121 & 0.0017 & 0.0027 & 0.0022 & 0.0014 & 0.0019 & 0.0020 \\
MH43 & 0.0030 & 0.0040 & 0.0035 & 0.0027 & 0.0024 & 0.0021 \\
NACA 642415 & 0.0039 & 0.0019 & 0.0014 & 0.0036 & 0.0011 & 0.0009 \\
\hline & 13 CPs & Weights & No Weights & 14 CPs & Weights & No Weights \\
\hline MH126 & 0.0049 & 0.0013 & 0.0016 & 0.0021 & 0.0007 & 0.0008 \\
MH121 & 0.0014 & 0.0020 & 0.0016 & 0.0014 & 0.0013 & 0.0016 \\
MH43 & 0.0027 & 0.0029 & 0.0025 & 0.0016 & 0.0020 & 0.0016 \\
NACA 642415 & 0.0031 & 0.0015 & 0.0010 & 0.0023 & 0.0007 & 0.0006 \\
\hline
\end{tabular}

3D coordinates are obtained according Section 2, and the fitting is applied. A circular section with a $1-\mathrm{m}$ diameter was placed over a cylinder with a radius of $5 \mathrm{~m}$ and was fitted using 14 control points as depicted in Fig. 7. The maximum distance of the B-spline to the 3D data points was $0.0002 \mathrm{~m}$, and the average distance was less than $0.0001 \mathrm{~m}$.

\section{Definition of the trailing edge}

When designing a turbine while considering its airfoils, the trailing edge of the airfoils is normally considered a single point to numerically calculate the lift and drag forces. This transforms the trailing edge of the sections into a sharp edge when defining the 3D geometry, which is often required in numerical calculations but is very fragile from a constructive perspective.

Airfoils can be trimmed at the trailing edge by reducing the chord by approximately $3 \%-5 \%$ or thickened by approximately $1 \%$ of the maximum thickness at this area without greatly altering its properties because the trailing edge is essentially maintained as sharp, so the fluid velocity at this part of the airfoil is high. The second method maintains an airfoil's chord.

These assumptions for the trailing edge produce two sharp edges and a ruled surface that encloses these two lines along the blade span. A smooth surface that joins these two edges and maintains the chord-length of the sections can be defined.

To create this surface, different curves that the surface will contain are needed. These curves (Fig. 8) are part of the trimmed airfoils and are constructed as follows:

1). Trim the data points according to a desired distance $d=3 \%-5 \%$ chord. $\boldsymbol{P}_{\mathbf{0}}$ and $\boldsymbol{P}_{\mathbf{3}}$ are the limit points of the data set.

2). Fit the trimmed data set according to Section 3 and obtain the B-spline $\boldsymbol{c}_{\boldsymbol{d}}$.

3 ). The tangent lines at the end points of the B-spline are $t_{0}$ and $t_{3}$ and follow the direction of the first and last leg of the control polygon of $\boldsymbol{c}_{\boldsymbol{d}}$. Calculate $d / 3$ to obtain the points $\boldsymbol{M}_{\mathbf{0}}$ and $\boldsymbol{M}_{\mathbf{3}}$ at the intersection of the tangent lines with the line parallel to $\boldsymbol{P}_{0} \boldsymbol{P}_{3}$ that is separated by $d / 3$ from the airfoil end $\boldsymbol{E}$. Note that $\boldsymbol{P}_{0} \boldsymbol{P}_{3}$ and $\boldsymbol{M}_{0} \boldsymbol{M}_{3}$ are perpendicular to the chord line.

4). The point $\boldsymbol{P}_{\mathbf{1}}$ that is symmetric to $\boldsymbol{P}_{\mathbf{0}}$ with respect to $\boldsymbol{M}_{\mathbf{0}}$, and the point $\boldsymbol{P}_{\mathbf{2}}$ that is symmetric to $\boldsymbol{P}_{\mathbf{3}}$ with respect to $\boldsymbol{M}_{\mathbf{3}}$ are the control points of a cubic Bézier curve that passes through $\boldsymbol{P}_{\mathbf{0}}$ and $\boldsymbol{P}_{3}$, is tangent at these points to $\boldsymbol{c}_{\boldsymbol{d}}$, and does not overpass the chord value because it is tangent to the line perpendicular to the chord line at $\boldsymbol{E}$.

The above-mentioned properties of the cubic Bézier curve that models the trailing edge come from an application of the Casteljau algorithm that defines Bézier curves. The final step of the proposed method is to create B-spline surfaces that contain the airfoils and their trailing edges.

\section{Lofting surface of the airfoils}

The last step of the method is the definition of B-spline surfaces that contain the previously defined B-splines with one surface for the trailing edges and a second surface for the rest of the airfoil curves.

The generalisation from curves to surfaces is not difficult due to the properties of B-splines, and thus, a lofting surface of the station pieces can be easily defined. The transition from spline curves to spline surfaces is achieved by turning the control polygon into a control net of control points $\boldsymbol{W}_{i j}\left(X_{i j}, Y_{i j}, Z_{i j}\right)$ using the same Bspline basis for the two parameters $u$ and $v$, as well as using two different lists of knots $\left\{u_{-1}, \ldots ., u_{N+n}\right\},\left\{v_{-1}, \ldots ., v_{M+m}\right\}$. 

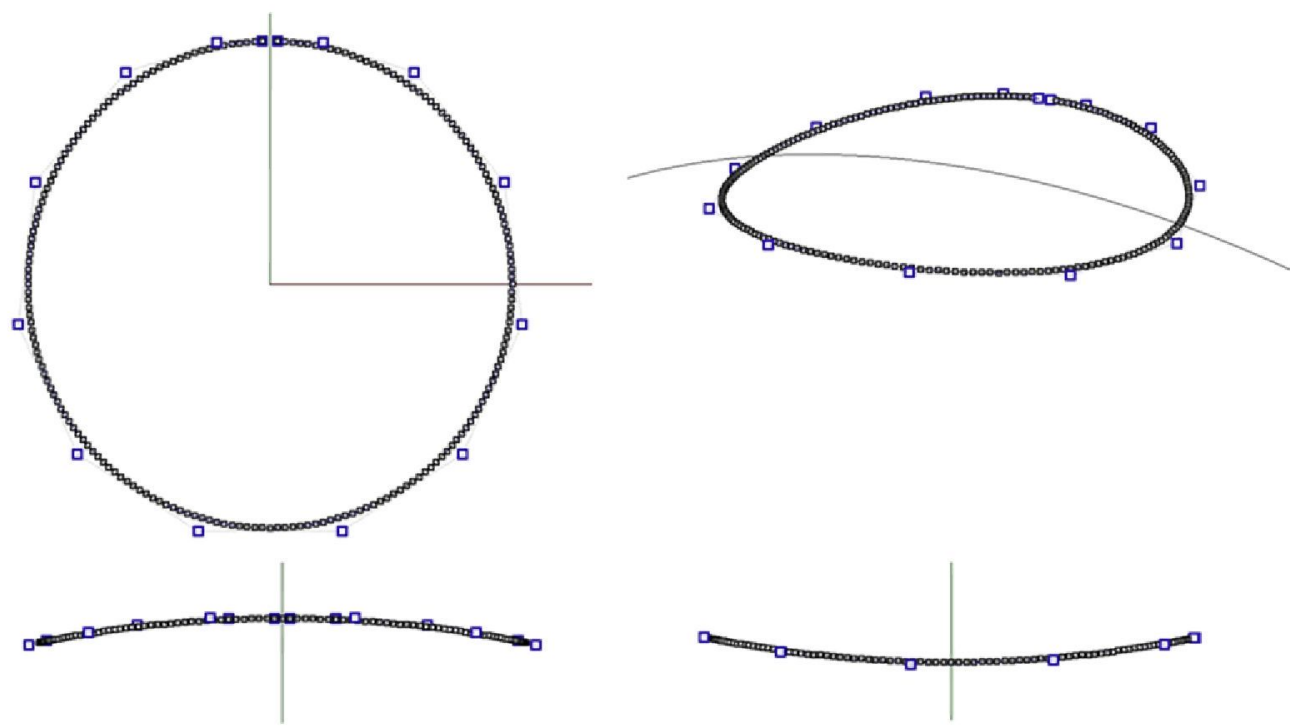

Fig. 7. Fitting of circular sections (root).

The lofting process of a set of $q$ B-splines (airfoils) with the same degree and list of knots is as follows. Find a B-spline surface $\boldsymbol{S}$ with degree $n$ by $m$ and $(N+1)$ by $(M+1)$ control points and a list of knots $\left\{u_{-1}, \ldots ., u_{N+n}\right\}$ and $\left\{v_{-1}, \ldots . ., v_{M+m}\right\}$ according to Eq. (7) that interpolates $q$ different B-splines $\boldsymbol{c}_{\boldsymbol{d}}(\boldsymbol{d}=0, \ldots, q-1)$ of $n$th degree with $N+1$ control points and a list of knots $\left\{u_{-1}, \ldots \ldots, u_{N+n}\right\}$ with the form of Eq. (8).

$\boldsymbol{S}(u, v)=\sum_{i=0}^{N} \sum_{j=0}^{M} \boldsymbol{W}_{i \mathfrak{i}} \cdot \mathrm{B}_{i}^{n}(u) \cdot \mathrm{B}_{j}^{m}(v)$

$\boldsymbol{c}_{\boldsymbol{d}}(u)=\sum_{i=0}^{N} \boldsymbol{V}_{\mathbf{i d}} \cdot \mathrm{B}_{i}^{n}(u) \quad(d=0, \ldots, q-\mathbf{1})$

Note that $\boldsymbol{V}_{\text {id }}$ are the control points of the different airfoils obtained in Section 3 and are expressed in matrix form. The values of $N, M$ and $q$ depend on user preferences regarding the definition of the blade surface. The interpolation can be written as:

$\boldsymbol{S}\left(u, v_{d}\right)=\sum_{i=0}^{N}\left(\sum_{j=0}^{M} \boldsymbol{W}_{i \mathbf{j}} \cdot \mathrm{B}_{j}^{m}\left(v_{d}\right)\right) \cdot \mathrm{B}_{i}^{n}(u)=\sum_{i=0}^{N} \boldsymbol{V}_{\mathbf{i d d}} \cdot \mathrm{B}_{i}^{n}(u)$

$$
=\boldsymbol{c}_{\boldsymbol{d}}(u) \quad(d=0, \ldots, q-1)
$$

This group of equations must be solved for a set of values of parameter $v_{d}(d=0, \ldots, q-1)$, which is called the choice of the

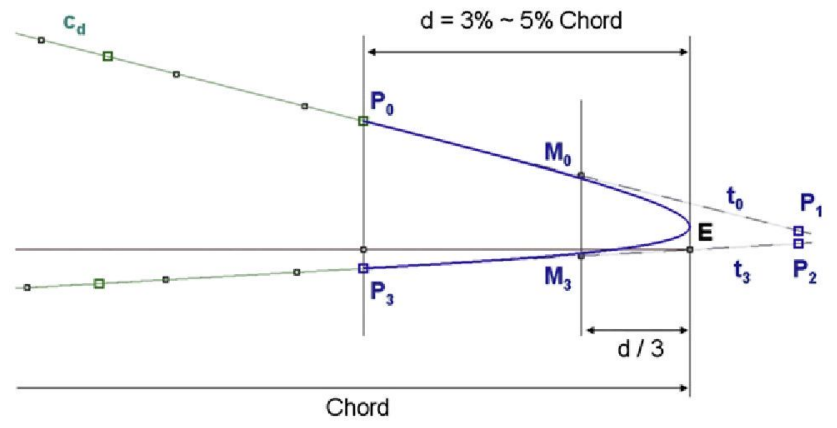

Fig. 8. Definition of a rounded trailing edge. parameterisation. Centripetal parameterisation produces good results for the shape of rotor blades. By identifying equal coefficients for each row in Eq. (9), $i=0, \ldots N$, the following linear system is obtained:

$\sum_{j=0}^{M} \boldsymbol{W}_{i j} \cdot \mathrm{B}_{j}^{m}\left(v_{d}\right)=\boldsymbol{V}_{\mathbf{i d}} \quad(d=0, \ldots, q-1)$

To obtain a unique solution for this system, $M+1=q$, where $q$ is the number of airfoils that define the blade. The $(M+1)$ by $(N+1)$ solutions are the control points $\boldsymbol{W}_{i j}$ of the lofting surface of Eq. (7) containing the airfoils.

\section{Examples}

Two different (marine current) turbines are presented in this section. The first example was defined to construct a scaled prototype of the turbine; therefore, its diameter is $0.5 \mathrm{~m}$. The airfoils used in the design were based on cambered Eppler856 profiles with 200 data points and a variable thickness/chord ratio along the blade span. The tolerance was $1.10^{-4} \mathrm{~m}$, and this value was achieved using cubic B-splines with 19 control points, considering the weight fitting detailed in Section 3. The root sections are circles modelled as B-splines with 19 control points as explained in the previously mentioned section. The hub was modelled as a frustum of cone.

In this example, the trailing edge was considered a straight segment between the first and the last point of the airfoils because a rounded end defined as in Section 5 could not be constructed for the prototype model due to its small size. In propeller/turbine models, the finishing of the blades' trailing edge requires manual labour.

The airfoils used for the modelling of Example 1 are shown in Fig. 9a). The details of some airfoils are shown in Fig. 9b), which shows the distribution of the 19 control points. A lofting surface of the B-splines is obtained according to Section 6, and the isoparametric curves of the blade surface are shown in Fig. 9c). A shaded view of the final blade is shown in Fig. 9d). The blade surfaces were used to construct a metal prototype of the rotor produced by a CNC machine directly from the surface information generated by applying the present method. 


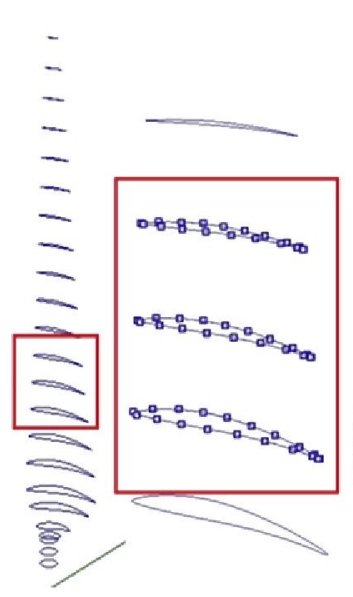

a

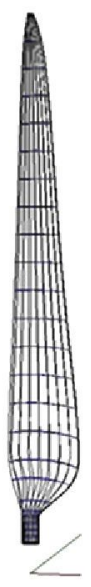

C

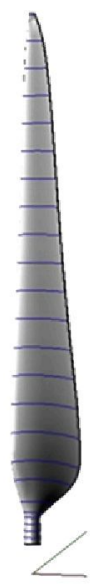

d

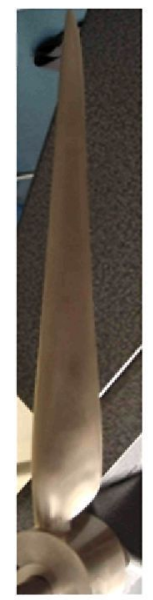

e

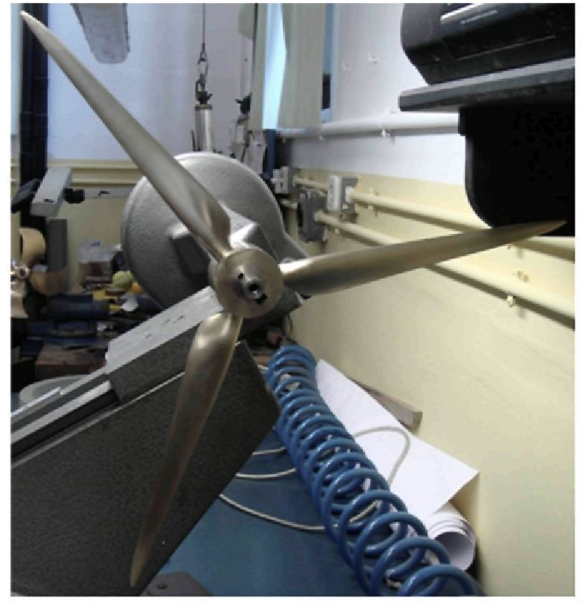

Fig. 9. Example 1.
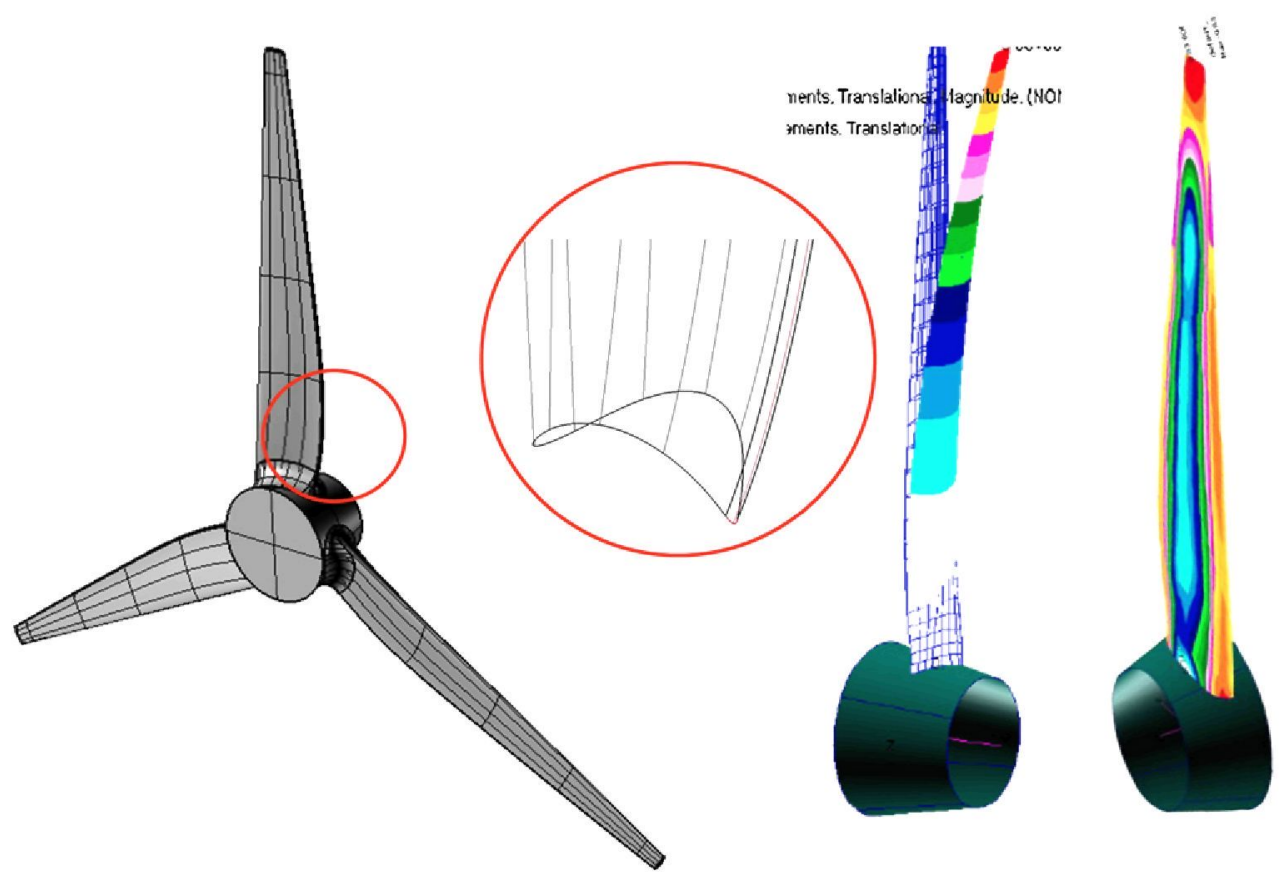

Fig. 10. Example 2.

The second example, shown in Fig. 10, presents a turbine project with a diameter of $1.7 \mathrm{~m}$ designed with Naca airfoils of variable thickness chord ratio. The tolerance value was selected as $1.10^{-3} \mathrm{~m}$, and this value was obtained using cubic B-splines of 12 control points and considering the weight fitting detailed in Section 3. In this case, the root sections were not circles because the turbine was a fixed-pitch turbine, and a fillet surface was created in the root area to reduce stress and strain near the hub. The blades presented a rounded trailing edge modelled according to Section 5, as shown in detail in Fig. 10. This Example 2 was used to compute FEA calculations before fabrication, directly with the surfaces generated by applying the method.

\section{Conclusions}

This paper has presented a practical method for producing a B-spline representation of the blades of a horizontal axis turbine. The method works with the information normally used in turbine blade design, such as airfoils data points and twist, rake and skew distributions, and produces a weighted fitting of the 3D airfoil data points allowing the leading edge, which has a major effect on the turbine properties, to be well reproduced.

The weighted least squares fitting also uses an iterative selection of the parameterisation, which allows for a given tolerance, which is very useful from a construction perspective. The method produced a blade surface with a minimum number of control points that fits the data points below a given allowance. This reduced number of control points permits a more simple surface definition, which is important in subsequent stages of the design process including numerical CFD calculations, FEA calculations or construction with $\mathrm{CNC}$ machines.

The weighted fitting was tested for airfoils with different characteristics and compared using various methods from the literature. It produced good results for the modelling of typical airfoils employed in turbine design. 
A B-spline definition of the sections' trailing edge was also produced such that the chord can be maintained without trimming or thickening the airfoils used during the initial design stage.

\section{Annex}

To introduce the notation for this paper, a brief discussion of Bspline curves follows. A B-spline curve is formed by several pieces of polynomial curves called Bézier pieces, and the entire curve is $\mathrm{C}^{2}$ (common curvature or second derivatives) at the junctions in the case of cubic B-splines. The curve is defined with a polygon called the control polygon and with an interpolation algorithm that allows for its construction by relating the curve to the control polygon. The interpolation steps are encoded in a family of piecewise polynomial functions $\mathrm{B}_{j}^{n}(u)$ called B-spline functions of the $n$th degree and are calculated with Cox de Boor's algorithm [14]. Cubic B-splines are the most frequently used curves in design.

A B-spline curve, $\boldsymbol{s}(\boldsymbol{u})$ in Eq. (10), is a linear combination of basis functions with $m+1$ control points $\boldsymbol{P}_{\boldsymbol{j}}$ as coefficients. Therefore, B-spline curves are parametric, $x=X(u), y=Y(u), z=Z(u)$, and the parameter $u$ is normally considered $[0,1]$. In the plane, $\boldsymbol{P}_{j}=\left(X_{j}, Y_{j}\right)$, $j=0, \ldots, m$ generate a B-spline $\boldsymbol{s}(\boldsymbol{u})$ of $n$th degree:

$s(u)=\sum_{j=0}^{m} P_{j} \cdot \mathrm{B}_{j}^{n}(u)=(X(u), Y(u))=\sum_{j=0}^{m}\left(X_{j} \cdot \mathrm{B}_{j}^{n}(u), Y_{\mathrm{j}} \cdot \mathrm{B}_{j}^{n}(u)\right)$

where the basis functions are obtained with Cox de Boor's algorithm in (11):

$$
\begin{aligned}
\mathrm{B}_{j}^{0}(u) & =\left\{\begin{array}{cc}
1 & u \in\left[u_{j-1}, u_{j}\right) \\
0 & u \notin\left[u_{j-1}, u_{j}\right)
\end{array} \quad \mathrm{B}_{j}^{n}(u)\right. \\
& =\frac{u-u_{j-1}}{u_{j+n-1}-u_{j-1}} \cdot \mathrm{B}_{j}^{n-1}(u)+\frac{u_{j+n}-u}{u_{j+n}-u_{j}} \cdot \mathrm{B}_{j+1}^{n-1}(u)
\end{aligned}
$$

The basis function $\mathrm{B}_{j}^{n}(u)$ depends on the knot vector $u_{j}$, which, in this work was chosen to be uniform with a multiplicity equal to the order of the curve at its ends, where the order is defined as the degree +1 . In this way, the B-spline interpolates the ends of its control polygon at $u=0$ and $u=1$ and is tangent at its ends to the first and last segment of its control polygon. This last property simplifies the mathematical definition of the curves used in the method. Note that the derivative $\boldsymbol{s}^{\prime}(\boldsymbol{u})$ of a B-spline is a linear combination of the derivatives of the basis functions:

$$
\begin{aligned}
\boldsymbol{s}^{\prime}(\boldsymbol{u}) & =\sum_{j=0}^{m} P_{j} \cdot \mathbf{B}_{j}^{\prime n}(\boldsymbol{u})=(X(\boldsymbol{u}), Y(\boldsymbol{u})) \\
& =\sum_{j=0}^{m}\left(X_{j} \cdot \mathbf{B}_{j}^{\prime n}(u), Y_{j} \cdot \mathbf{B}_{j}^{m}(u)\right)
\end{aligned}
$$

When the number of control points $(m+1)$ is equal to the order of the curve $(n+1)$, then the B-spline curve is formed from just one piece and can be called a Bézier curve of the $n$th degree. In this work, all of the curves are referred to as B-splines for the sake of clarity. A variable presented in bold letters indicates that this element is formed from several components, such as points, vectors or B-spline curves that have $X, Y$ and $Z$ components.

[1] Piegl L. Modifying the shape of rational b-spline. Part 2: surfaces. CAD 1989; 21:538-46.

[2] Au C, Yuen M. Unified approach to NURBS curve shape modification. CAD 1995;27:85-93.

[3] Woodward C. Cross-sectional design of B-spline surfaces. Comput Graphics 1987;11:193-201.

[4] Piegl LA, Tiller W. Cross-sectional design with boundary constraints. Eng Comput 1999;1999(15):171-80.

[5] Piegl LA, Tiller W. In: The NURBS Book. Springer; 1997. p. 410-3.

[6] Hoscheck J, Müller R. Turbine blade design by lofted B-spline surfaces. Computational Appl Math 2000;119:235-48.

[7] Bentamy A, Guibault F, Trépanier JY. Cross-sectional design with curvature constraints. CAD 2005;37:1499-508.

[8] Mauclère X. Automatic 2D airfoil Generation, Evaluation and optimisation using MATLAB and XFOIL. Master thesis. Technical University of Denmark, 2009

[9] Hansen MOL. In: Aerodynamics of wind turbines. Earthscan; 2008.

[10] Burton T, Sharpe D, Jenkins N, Bossanyi E. In: Wind energy handbook. Wiley; 2001.

[11] Spera DA. In: Wind turbine technology: fundamental concepts of wind turbine engineering. The American Society of Mechanical Engineers; 1998.

[12] Jenkins A, Traub J. A three-stage variable-shift iteration for polynomial zeros and its relation to generalized Rayleigh iteration. Numerische Mathematik $1970: 14 / 3: 252-63$.

[13] Fuglsang P, Bak C. Status of the RISø Wind Turbine Airfoils, ln Proceedings of the European Wind Energy Conference, 16-19 June 2003 Madrid, 2003.

[14] Farin G. Curves and surfaces for CAGD. 5th ed. San Francisco: Morgan Kaufmann; 2001. 